\title{
Modelación del ruido producido por tránsito vehicular en la ciudad de Guatemala
}

\author{
Modeling of noise produced by \\ vehicular traffic in Guatemala City
}

\section{Como citar el artículo}

Sánchez Girón, A. I. ., \& Urrutia Campo, M. A. . Modelación del ruido producido por tránsito vehicular en la ciudad de Guatemala . Revista Naturaleza, Sociedad Y Ambiente, 8(1). DOI: https://doi.org/10.37533/cunsurori.v8i1.60

Ana Isabel Sánchez Girón y Marco Antonio Urrutia Campo

Municipalidad de Guatemala

Recibido: 20 de abril de 2021 / Aceptado: 18 de junio de 2021

Disponible en internet el 10 de octubre de 2021

*Autor para correspondencia, correo electrónico: aisanchez@muniguate.com

\section{Resumen}

Los mapas de ruido son una herramienta de planificación para las ciudades que se enfrentan a problemas que afectan el bienestar de la población e implican daños al ambiente, como lo es la contaminación acústica. Este tipo de contaminación requiere acciones adecuadas para su manejo ante los retos que se presentan por el rápido crecimiento de la población y otros factores económicos y sociales. Los cuales se deben tomar en consideración para construir una ciudad sostenible, por lo que se hace necesario evaluar las distintas fuentes que generan este tipo de contaminación. Por esta razón es que se ha realizado una modelación del ruido en la ciudad de Guatemala con el objetivo de determinar, a través de mapas, las zonas más afectadas por problemas de contaminación acústica por emisiones provenientes de fuentes móviles (tránsito vehicular) a través del uso del software SoundPLAN. Con base en este estudio se concluyó que en los tres tramos viales analizados se presenta en promedio un nivel de ruido diurno mayor de $65 \mathrm{~dB}$ y en la noche un nivel superior a los $55 \mathrm{~dB}$, superando así los límites permisibles establecidos para cada uso del territorio según la normativa de referencia, Ley española 37/2003.

Palabras clave: Ruido, mapas de ruido, contaminación acústica, tránsito vehicular, SoundPLAN

\begin{abstract}
Noise maps are a planning tool for cities that face problems that affect the well-being of the population and involve damage to the environment, such as noise pollution. This type of contamination requires adequate actions for its management in the face of the challenges presented by the rapid growth of the population and other economic and social factors. Which must be taken into consideration to build a sustainable city, so it is necessary to evaluate the different sources that generate this type of pollution. For this reason, a noise modeling has been carried out in Guatemala City in order to determine, through maps, the areas most affected by problems of noise pollution from emissions from mobile sources (vehicular transit) through the use of SoundPLAN software. Based on this study, it was concluded that in the three analyzed road sections there is an average daytime noise level greater than $65 \mathrm{~dB}$ and at night a level greater than $55 \mathrm{~dB}$, thus exceeding the permissible limits established for each use of the territory. according to the reference regulations, Spanish Law 37/2003.
\end{abstract}

Keywords: Noise, noise maps, noise pollution, vehicular traffic, SoundPLAN 


\section{Introducción}

El ruido es definido por la Organización Mundial de la Salud como todo aquel sonido no deseado (OMS, 1999). También se puede definir como aquel sonido que es percibido por quien lo escucha como molesto o irritante.

La exposición al ruido afecta el bienestar y además según la OMS puede tener efectos perjudiciales en la salud como: deficiencia auditiva, trastornos del sueño y enfermedades cardiovasculares: hipertensión y cardiopatía por exposición prolongada. Así mismo la OMS ha identificado que las principales fuentes de ruido son urbanas y provienen de actividades de ocio, comerciales, constructivas, por tránsito de vehículos, entre otras. Es por esto que la presencia del ruido se ha considerado como un tipo de contaminación ambiental denominada acústica.

A nivel de país en las áreas urbanas es donde se puede presentar este tipo de contaminación, que según datos del Ministerio de Ambiente y Recursos Naturales (MARN) del 2010 al 2014 se han recibido 755 denuncias por ruido y según mediciones efectuadas por esta institución existen sitios donde se superan los 100 decibeles (dB) lo cual es perjudicial para la salud siendo 65 decibeles la intensidad máxima que puede soportar el oído humano según la OMS (Pitán, 2015).

Específicamente son las ciudades las que se caracterizan por presentar una gran cantidad de fuentes de ruido por las actividades que en ella se realizan. La ciudad de Guatemala no es la excepción. Esta situación representa un reto para la administración municipal en cuanto a su gestión porque no se cuenta con una normativa que regule la contaminación acústica solamente la contaminación audial generada por sonido amplificado según el acuerdo municipal COM-2-2012 para la ciudad de Guatemala.

Actualmente se cuenta con herramientas como los mapas de ruido que permiten evaluar los niveles de ruido procedentes del tránsito vehicular o la industria. Los datos que se obtienen permiten entonces realizar un diagnóstico de la situación actual que pueden ser utilizados para tomar medidas de mitigación, orientar la planificación territorial y establecer los límites permisibles.

\section{Referente Teórico}

Los mapas de ruido son una representación gráfica de los niveles de presión sonora para un sitio en específico. Esta herramienta permite evaluar a priori la eficacia de acciones correctoras, en el corto o largo plazo, así como estimar el número de personas expuestas al ruido (CAEND, 2011).

En Europa la directiva 2002/49/CE sobre evaluación y gestión del ruido ambiental exige la elaboración de estos para las ciudades, así como su actualización cada 5 años o antes si existen cambios en la estructura urbana que sean significativos en la emisión de niveles de ruido.

Para la elaboración de los mapas de ruido existen dos formas: por muestreo y por simulación (AISTEC, s.f). En este caso se hace uso del método por simulación que presenta la ventaja de facilidad de cálculo, consideración de escenarios actuales y a futuro, análisis de diferentes fuentes, la interacción de estas con el ambiente acústico. 
En este estudio se utiliza el software de predicción denominado SoundPLAN. El cual está destinado para la modelación del ruido. Fue creado en Alemania y se utiliza desde 1986. Este software puede modelar el ruido del tránsito vehicular, el ruido ocupacional en interiores y exteriores, el ruido industrial en general, el ruido de los aviones y la acústica de cuartos (GeoNoise, s.f).

SoundPLAN es un software con estructura modular. Los módulos dependen del tipo de evaluación de ruido que se pretende realizar, en este caso, para la modelación de ruido por tránsito se utilizan los siguientes módulos: Base de datos geográfica, Caja de herramientas, Mapa de fachadas, Mapa de ruido de cuadrícula, Cartografía y Propagación de Ruido de Carreteras.

- Base de datos geográfica (Geo-base de datos): en esta base de datos se ingresan los datos geométricos denominados objetos. Los cuales forman los geo-archivos que en conjunto se denominan situaciones. Desde este módulo es posible obtener una vista del modelo de objeto sólido 3D y crear archivos como barreras acústicas, puntos, entre otros. Además, permite conectarse a Google Maps, Google Earth, OSM y WMS Server para descargar información.

- Caja de herramientas: en este módulo se pueden generar los mapas de ruido y las zonas de conflicto acústico, en los que se superan los valores límite (AACUSTICA, s.f.).

- Mapa de fachadas: este módulo permite calcular el nivel de sonido incidente a lo largo de las fachadas de los edificios.
- Mapa de ruido de cuadrícula: este módulo calcula el nivel de presión acústica sobre una rejilla equidistante y gráficamente presenta la salida en forma de datos de cuadrícula o como curvas de nivel de ruido (SoundPLAN, s.f.). Los resultados se pueden exportar a formatos de archivo vectorial Shapefile.

- Cartografía: en este módulo se pueden generar mapas y personalizarlos de forma gráfica.

- $\quad$ Propagación de Ruido de Carreteras: controla qué foco de ruido puede ser utilizado y qué normativas están disponibles para los cálculos (AACUSTICA, s.f.). Entre las normativas para cálculo de ruido por tránsito urbano disponibles en este módulo destacan las que se muestran en la tabla No. 1.

Tabla No. 1

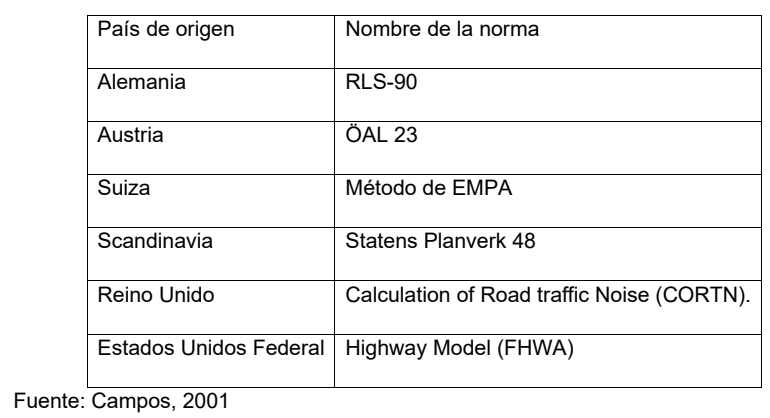

La normativa utilizada en este estudio es la RLS 90 de Alemania para la predicción de ruido por tránsito en carreteras y parqueos. RLS-90 usa el método de fuente puntual con divergencia, atenuación de terreno, apantallamientos y reflexiones (Peña, 2012).

El modelo RLS-90 de emisión a nivel de fuentes toma en cuenta las características del flujo de tráfico, velocidad de los vehículos, tipo de vehículos y textura de la carretera (Mishra, A. R., Mishra, R. K., \& Singh, A., 2019). 
El descriptor utilizado en este modelo es el Leq1hora y también entrega resultados para rangos de tiempos mayores: para el día (6:00 AM a 10:00 PM) y la noche (10:00 PM a 6:00 AM). Estos rangos de tiempo expresan los niveles de ruido para el índice acústico en un periodo evaluación diurno (6:00 AM a 10:00 $P M)$, Lday, y para el periodo nocturno (10:00 PM a 6:00 AM), Lnight.

El índice de ruido asociado al día establece el nivel sonoro medio para el periodo diurno y el índice de ruido asociado a la noche es un indicador del nivel sonoro durante la noche. Los resultados expresados por medio de estos índices permiten comparar con los niveles permitidos. En Guatemala no existe un reglamento específico que establezca los límites permitidos para el ruido generado por fuentes diferentes a megáfonos, equipos de sonido expuestos al público o vehículos con aparatos reproductores de sonido.Por lo que para este estudio se toma como referente comparativo una normativa internacional, la Ley española 37/2003 del Ruido. La cual establece objetivos de calidad acústica aplicables a diferentes áreas.

En la tabla No. 2 se muestran los valores de los índices acústicos, fijados por esta ley, que no deben superarse para el cumplimiento de los objetivos de calidad acústica en áreas urbanizadas existentes.

Tabla No. 2 Niveles máximos permisibles de ruido en áreas urbanizadas

\begin{tabular}{|c|c|c|c|c|}
\hline & \multirow{2}{*}{ Tipo de área acústica } & \multicolumn{3}{|c|}{ índices de ruido } \\
\hline & & Ld & Le & Ln \\
\hline e & $\begin{array}{l}\text { Sectores del territorio con predominio de } \\
\text { suelo de uso sanitario, docente y } \\
\text { cultural que requiera una especial } \\
\text { protección contra la contaminación } \\
\text { acústica }\end{array}$ & 60 & 60 & 50 \\
\hline A & $\begin{array}{l}\text { Sectores del territorio con predominio de } \\
\text { suelo de uso residencial. }\end{array}$ & 65 & 65 & 55 \\
\hline D & $\begin{array}{l}\text { Sectores del territorio con predominio de } \\
\text { suelo de uso terciario distinto del } \\
\text { contemplado en c). }\end{array}$ & 70 & 70 & 65 \\
\hline C & $\begin{array}{l}\text { Sectores del territorio con predominio de } \\
\text { suelo de uso recreativo y de } \\
\text { espectáculos }\end{array}$ & 73 & 73 & 63 \\
\hline B & $\begin{array}{l}\text { Sectores del territorio con predominio de } \\
\text { suelo de uso industrial }\end{array}$ & 75 & 75 & 65 \\
\hline$f$ & $\begin{array}{l}\text { Sectores del territorio con predominio de } \\
\text { suelo de uso industrial }\end{array}$ & $\begin{array}{l}\text { Sin } \\
\text { determinar }\end{array}$ & $\begin{array}{l}\text { Sin } \\
\text { determinar }\end{array}$ & $\begin{array}{l}\text { Sin } \\
\text { determinar }\end{array}$ \\
\hline
\end{tabular}




\section{Descripción del área de estudio}

Los tramos viales analizados se encuentran ubicados en el municipio de Guatemala. Este municipio se caracteriza por ser un área predominantemente urbana en la que se concentran las principales actividades económicas, sociales y culturales del país por tratarse de la ciudad capital. Es por esto que se ve afectada principalmente por problemas derivados del tránsito vehicular por el parque vehicular propio y el proveniente de otros municipios.

Derivado de esto la Dirección de Movilidad Urbana de la Municipalidad de Guatemala ha realizado conteos para determinar la cantidad de vehículos que transitan sobre los principales accesos a la ciudad. Con base en la ubicación de estos sitios se seleccionaron los tramos para el estudio, en total tres, que se describen en la tabla No. 3 y se pueden visualizar en la figura No. 1.

Tabla No. 3

\begin{tabular}{|l|l|l|}
\hline & Tramo & Ubicación \\
\hline 1 & Kilómetro 15.5 al 18 & zona 18 \\
\hline 2 & Kilómetro 4.5 al 6.5 & zona 18 \\
\hline 3 & Bulevar la pedrera & zona 6 \\
\hline
\end{tabular}

Figura No. 1 Tramos analizados

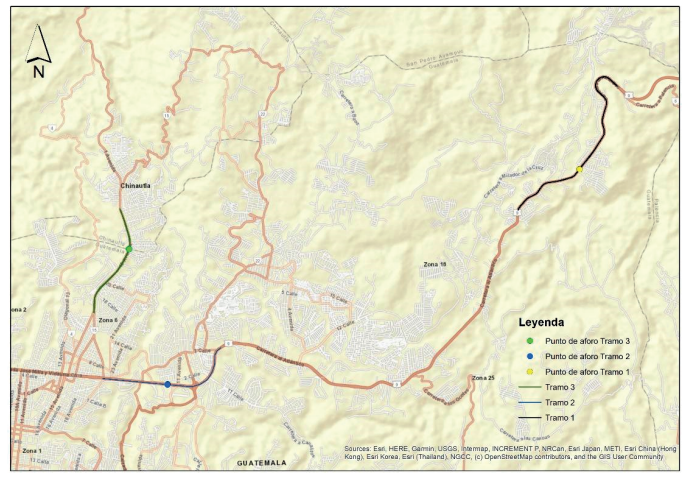

Fuente: elaboración propia

\section{Metodología}

La técnica utilizada para este estudio es cuantitativa. Se prepararon los datos de entrada: edificios, el área de ocupación por habitante, el aforo vehicular y las características viales para cada tramo.

Los datos de los edificios o huella de construcción, consisten en un archivo vectorial tipo shapefile elaborado por la Dirección de Catastro y Administración del IUSI de la Municipalidad de Guatemala, donde se encuentran ubicadas vectorizadas las edificaciones con el número de niveles.

El área de ocupación en metros cuadrados por habitante se determinó para las zonas donde se encuentran ubicados los tramos, los resultados se muestran en la tabla No. 4.

Tabla No. 4 Área de ocupación por habitante

\begin{tabular}{|l|l|l|l|}
\hline Zona & Área $\left(\mathrm{km}^{2}\right)$ & Población & Área de ocupación por habitante $(\mathrm{m} 2 /$ habitante $)$ \\
\hline 18 & 43.66 & 205204 & 212.75 \\
\hline 6 & 6.66 & 70778 & 94.04 \\
\hline
\end{tabular}

Fuente: Elaboración propia con información del Censo del INE 2018

Las características viales de los tramos identifican aspectos relacionados con las dimensiones (longitud, ancho de carriles y camellones), velocidad promedio y tipo de revestimiento.

Tabla No. 5 Características viales de los tramos analizados

\begin{tabular}{|l|l|l|l|l|l|l|}
\hline Tramo & $\begin{array}{l}\text { Tipo de } \\
\text { superficie } \\
\text { (revestimiento) }\end{array}$ & $\begin{array}{l}\text { Cantidad } \\
\text { de } \\
\text { carriles }\end{array}$ & $\begin{array}{l}\text { Ancho } \\
\text { de carril } \\
\text { derecho } \\
(\mathrm{m})\end{array}$ & $\begin{array}{l}\text { Ancho de } \\
\text { carril } \\
\text { izquierdo } \\
(\mathrm{m})\end{array}$ & $\begin{array}{l}\text { Velocidad } \\
\text { periodo } \\
\text { diurno } \\
(\mathrm{km} / \mathrm{h})\end{array}$ & $\begin{array}{l}\text { Velocidad } \\
\text { periodo } \\
\text { nocturno } \\
(\mathrm{km} / \mathrm{h})\end{array}$ \\
\hline 1 & Concreto & 4 & 3.75 & 3.75 & 50 & 60 \\
\hline 2 & Concreto & 4 & 3.3 & 3.3 & 50 & 60 \\
\hline 3 & Asfalto & 4 & 3.75 & 3.75 & 50 & 60 \\
\hline
\end{tabular}


Los datos de aforo indican la cantidad promedio de vehículos que transitan por hora, clasificados en livianos y pesados, para dos periodos: diurno (6:00-22:00 horas) y nocturno (22:00-6:00 horas).

Tabla No. 6 Cantidad de vehículos por hora para cada tramo

\begin{tabular}{|l|l|l|l|l|}
\hline \multirow{2}{*}{} & \multicolumn{4}{|l|}{ Cantidad de vehículos por hora (vehículos/hora) } \\
\cline { 2 - 5 } & \multicolumn{2}{|l|}{ Liviano } & \multicolumn{2}{l|}{ Pesado } \\
\hline Tramo & Día & Noche & Día & Noche \\
\hline 1 & 1361 & 1591 & 330 & 145 \\
\hline 2 & 3866 & 4355 & 557 & 392 \\
\hline 3 & 1668 & 2322 & 115 & 126 \\
\hline
\end{tabular}

Fuente: elaboración propia

Con esta información se inició con la modelación en el software SoundPLAN versión 8.2 a través de sus diferentes módulos. Estableciendo en primer lugar el modelo a utilizar, así como los periodos de evaluación. El modelo seleccionado en este caso es el RLS-90 y los periodos son dos: 22:00-6:00 horas (diurno) y de 6:00-22:00 horas (nocturno).

Posteriormente se ingresó y preparó la información en Geo-base de datos. Donde se creó la situación a evaluar y los ficheros para cada componente: carretera, edificios y elevación. El archivo vectorial de los edificios se importó desde el archivo en la Geo-base de datos. Los archivos vectoriales de carreteras y elevación se importaron desde OpenStreetmap, servicio de mapas online, con la opción conexión a OSM.

La información de los edificios se ingresó desde la opción preparar edificios y aquí se indicó la longitud mínima de un edificio (1 metro) y el área de ocupación por habitante. Con estos datos se delimitó el área de cálcu- lo y se ajustaron los objetos al modelo de elevación digital con la herramienta Set to DGM. Para verificar que toda la información se ingresó de manera correcta se ejecutó la opción de preflight, que notifica en caso de errores.

Finalmente se procesó la información en el módulo de cálculo para generar el mapa de ruido, fachadas y conflicto acústico utilizando un tamaño de malla de 15 metros. El mapa de ruido y de fachadas se generó con la opción ruido exterior. El mapa de conflicto acústico se trabajó especificando los límites de ruido permitidos para día y noche con la herramienta zona de conflicto.

Gráficamente los resultados se visualizan en el módulo cartografía, donde fueron editados para presentarlos y posteriormente exportarlos como imagen y archivo vectorial tipo shapefile.

\section{Resultados y discusión}

9.1 Mapa de ruido para los índices acústicos diurnos, Lday, y nocturnos, Lnight

Los mapas de ruido generados muestran cómo están distribuidos los niveles de ruido para los índices acústicos diurnos y nocturnos en cada uno de los tramos analizados. Los mayores niveles de decibeles se generan desde la carretera y disminuyen conforme se alejan de este foco de emisión. Lo cual puede visualizarse claramente para el tramo 1 y 3 . En el caso del tramo 2 esta distribución se ve afectada por la ubicación de las edificaciones alrededor.

Los mapas de ruido diurno y nocturno para el tramo 1 se muestran en la figura No. 2. Para el periodo diurno en la carretera y sus alrededores el nivel alcanzado es de hasta 65 deci- 
beles ocupando una superficie de $0.77 \mathrm{Km} 2$, en el caso del periodo nocturno se mantiene ese mismo nivel, pero el área es menor, 0.64 $\mathrm{Km} 2$. Las edificaciones afectan la propagación de las ondas acústicas, actuando como barreras, de forma que en estos sitios los

niveles de ruido disminuyen a una menor distancia a comparación de aquellos lugares donde no hay edificios.

Figura No. 2 Mapa de ruido diurno y nocturno para el tramo 1
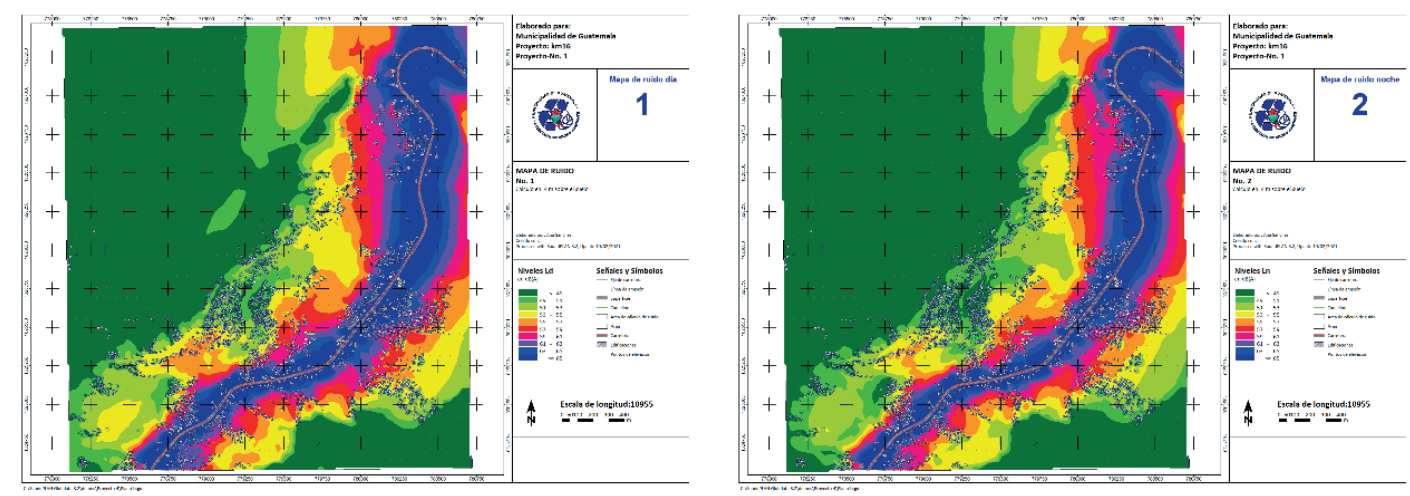

Fuente: elaboración propia, empleando SoundPLAN.

Los mapas para el tramo 2 se muestran en la figura No. 3. Donde para ambos periodos y tanto en la carretera como en sus alrededores se mantienen valores iguales o superiores a los 100 decibeles. Presentándose una zona en ambos periodos, de $0.02 \mathrm{Km} 2$ en promedio, donde se alcanzan hasta los 130 decibeles y en la que las edificaciones se encuentran más alejadas. Cabe resaltar que en este tramo transita la mayor cantidad de vehículos por hora, tanto livianos como pesados.

Figura No. 3 Mapa de ruido diurno y nocturno para el tramo 2
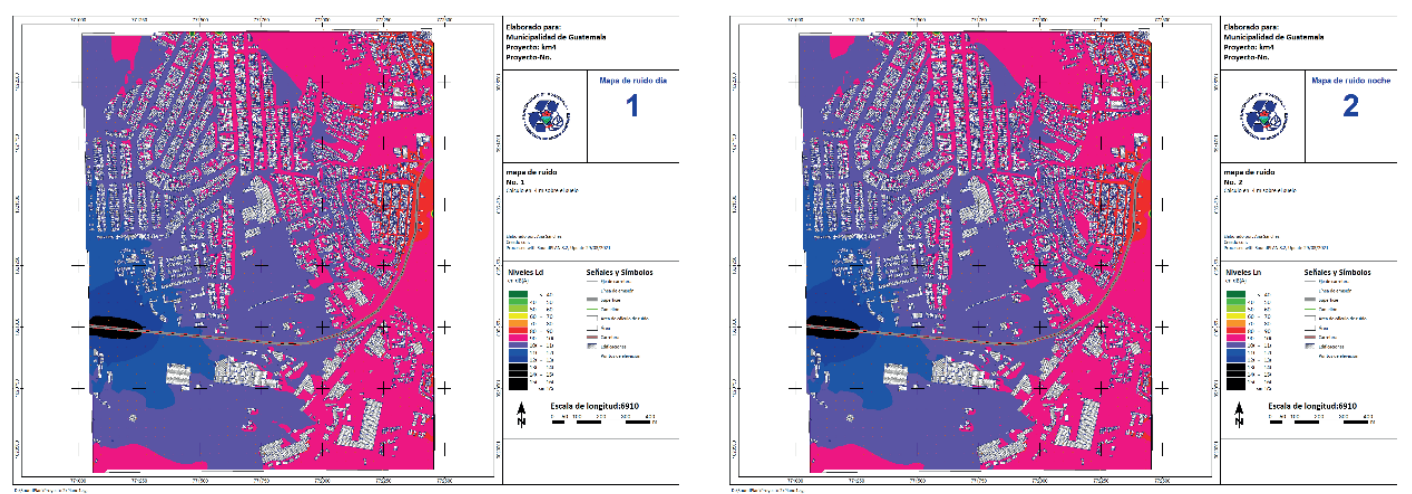

Fuente: elaboración propia, empleando SoundPLAN. 
Para el tramo 3 los mapas de ruido diurno y nocturno se muestran en la figura No. 4. Desde la carretera para ambos periodos los valores de ruido alcanzan un rango entre los 70 y 80 decibeles, disminuyendo con la distancia y la presencia de edificaciones. A comparación del tramo 1 la cantidad de vehículos pesados que transita por hora es mayor en este tramo.
Cabe resaltar que en este tramo se encuentra ubicado un estadio y una industria dedicada a la fabricación de cemento, esta última aporta con la cantidad de transporte pesado que transita en la zona. Tanto la industria como el estadio no se consideraron como fuentes puntuales de emisión en la modelación por no ser el fin del estudio, pero que se deben tomar en cuenta para una evaluación general del ruido.

Figura No. 4 Mapa de ruido diurno y nocturno para el tramo 3
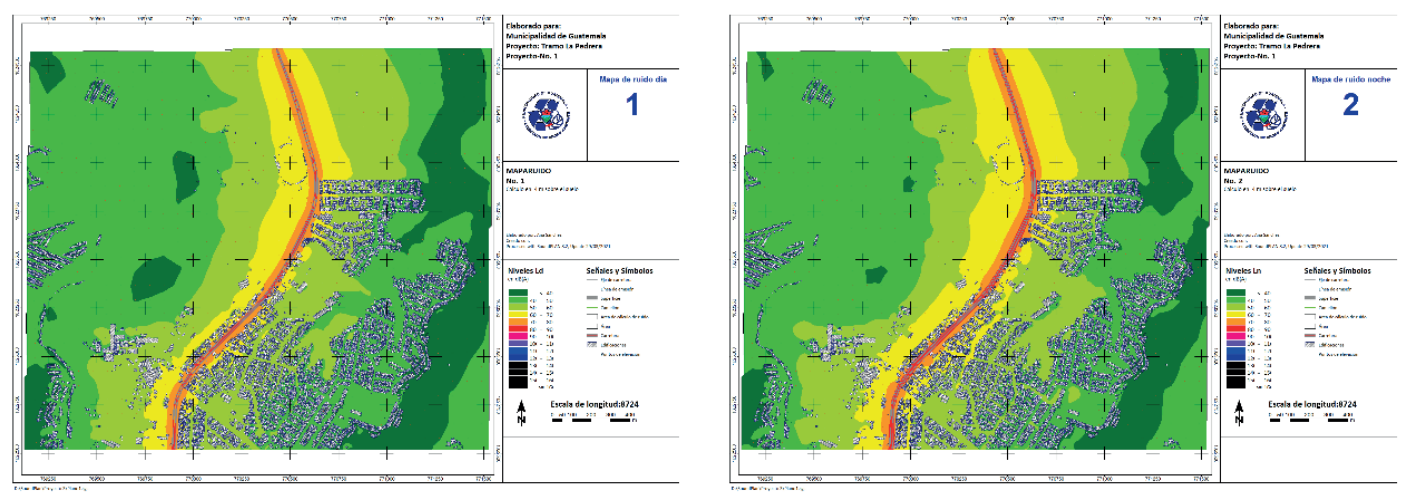

Fuente: elaboración propia, empleando SoundPLAN.

Mapa de fachadas para los índices acústicos diurnos, Lday, y nocturnos, Lnight

Con el mapa de fachadas se determinó a qué nivel de presión sonora están expuestas las fachadas de las edificaciones. Este cálculo se realizó para para el primer nivel de cada edificio.

Resultando, como se muestra en las figuras 5,6 y 7 , que las fachadas de las edificaciones que están expuestas a niveles más altos de ruido son las que están paralelas y próximas al sentido del tránsito vehicular, mientras que las fachadas no expuestas directamente presentan niveles más bajos de ruido ya que las demás paredes mitigan el efecto de propagación de las ondas acústicas.

En el primer tramo 3,560 fachadas de edificios están expuestas a más de $65 \mathrm{~dB}$ en el día y en la noche un total de 14,799 están expuestas a más de $55 \mathrm{~dB}$.

Figura No. 5 Mapa de fachada diurno y nocturno para el tramo 1
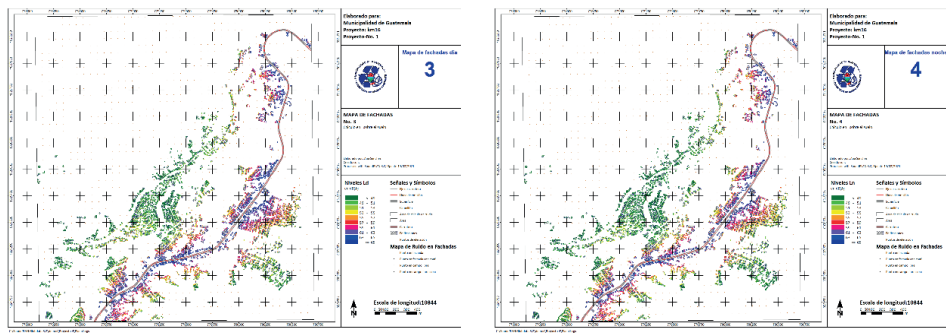

Fuente: elaboración propia, empleando SoundPLAN. 
En el segundo tramo 39,392 fachadas de el día y esa misma cantidad de edificios en la edificios están expuestas a más de $70 \mathrm{~dB}$ en noche está expuesta a más de $65 \mathrm{~dB}$.

Figura No. 6 Mapa de fachada diurno y nocturno para el tramo 2
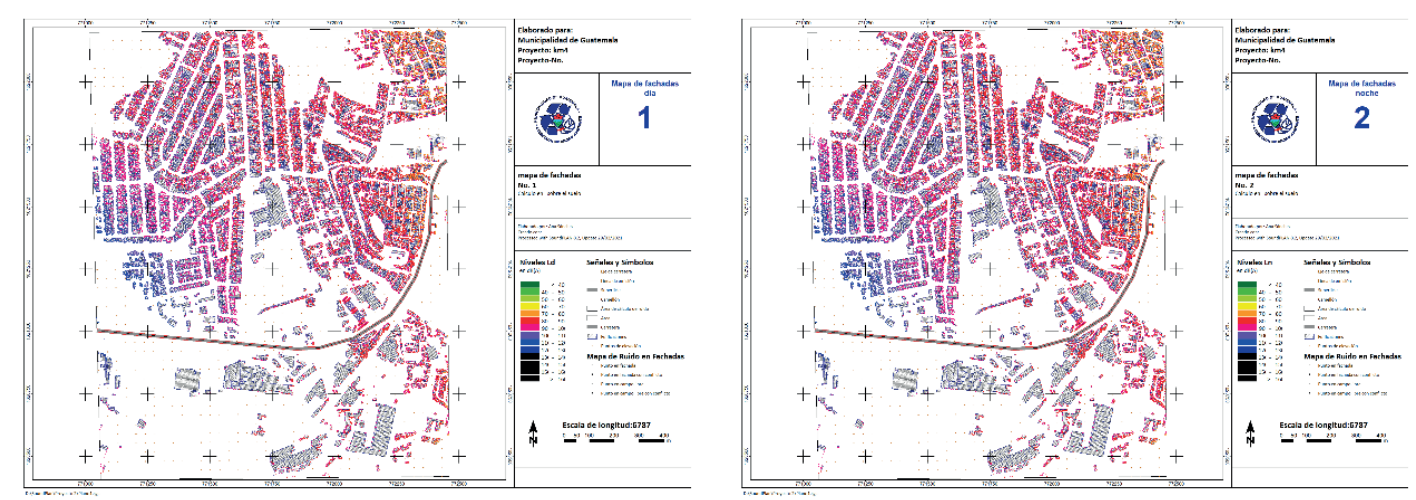

Fuente: elaboración propia, empleando SoundPLAN.

En el tercer tramo 313 fachadas de edificios están expuestas a más de $65 \mathrm{~dB}$ en el día y en la noche un total de 1,549 están expuestas a más de $55 \mathrm{~dB}$.

Figura No. 7 Mapa de fachada diurno y nocturno para el tramo 3
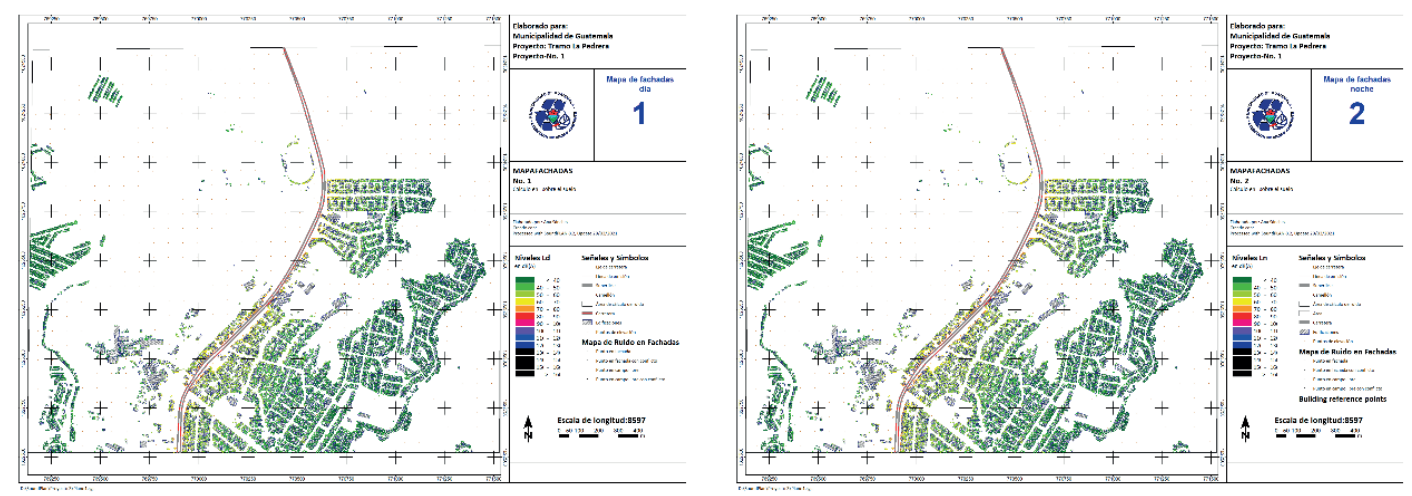

Fuente: elaboración propia, empleando SoundPLAN. 
Mapas de conflicto acústico para los índices acústicos diurnos, Lday, y nocturnos, Lnight.

Por medio de los mapas de conflicto acústico se pudieron identificar las zonas en las que se superan los límites establecidos por la normativa de referencia, Ley española $37 / 2003$.
Para el tramo 1, ver figura No. 8, el área se considera como predominantemente residencial por lo que los valores máximos permitidos para el día son $65 \mathrm{~dB}$ y para la noche 55 $\mathrm{dB}$. La superficie en la que no se cumple con este límite es de $0.97 \mathrm{Km} 2$ por el día y de 2.1 $\mathrm{Km} 2$ por la noche.

Figura No. 8 Mapa de conflicto acústico diurno y nocturno para el tramo 1
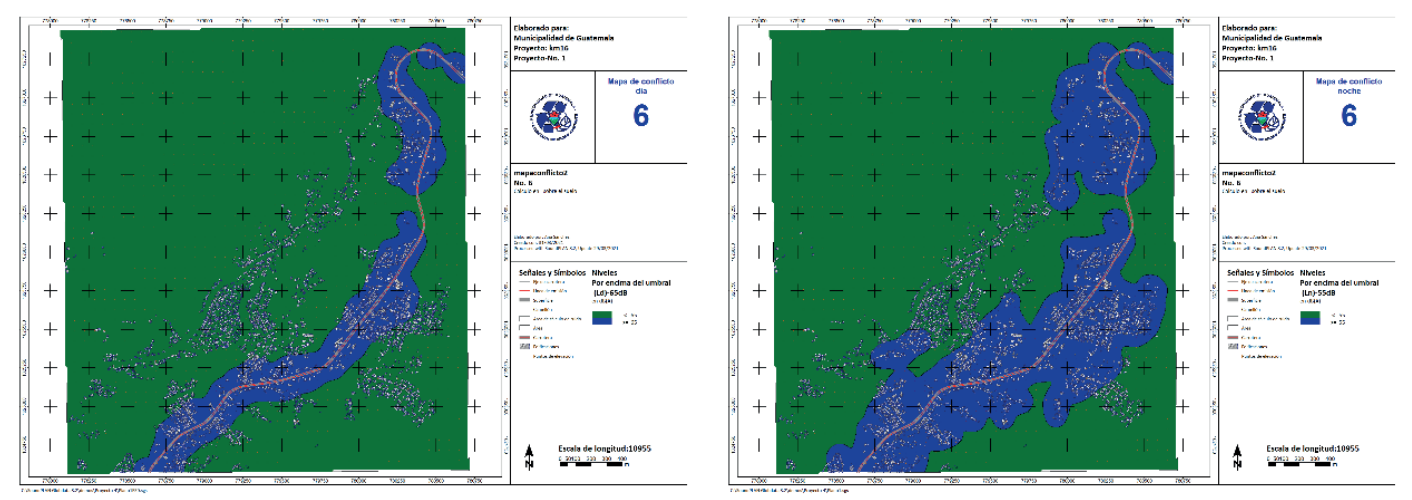

Fuente: elaboración propia, empleando SoundPLAN.

Para el tramo 2, ver figura No. 9, el área se considera de uso predominantemente comercial por lo que los valores máximos permitidos corresponden a una categoría de sector de uso terciario con $70 \mathrm{~dB}$ para el día y $65 \mathrm{~dB}$ para la noche. La superficie en la que no se cumple con este límite es de $2.25 \mathrm{Km} 2$ para el día y de $2.27 \mathrm{Km} 2$ para la noche.

Figura No. 9 Mapa de conflicto acústico diurno y nocturno para el tramo 2
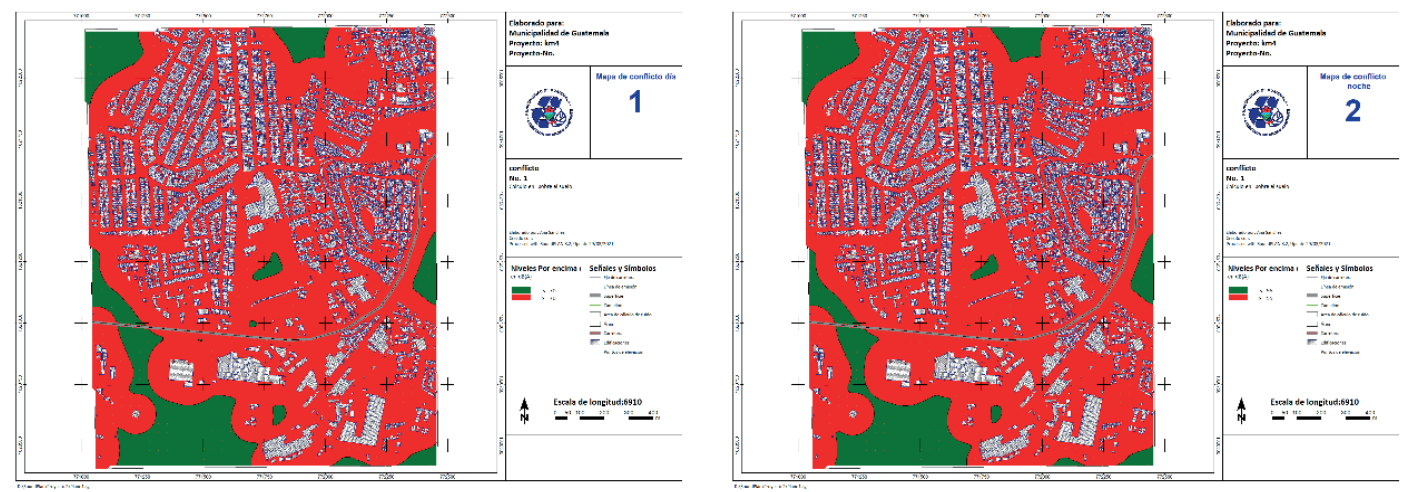

Fuente: elaboración propia, empleando SoundPLAN. 
Para el tramo 3, ver figura No. 10, el área se considera como un predominantemente residencial con valores máximos permitidos de $65 \mathrm{~dB}$ para el día y $55 \mathrm{~dB}$ para la noche. La superficie en la que no se cumple con este límite es de $1.03 \mathrm{Km} 2$ para el día y de 2.46 $\mathrm{Km} 2$ para la noche.

Figura No. 10 Mapa de conflicto acústico diurno y nocturno para el tramo 3
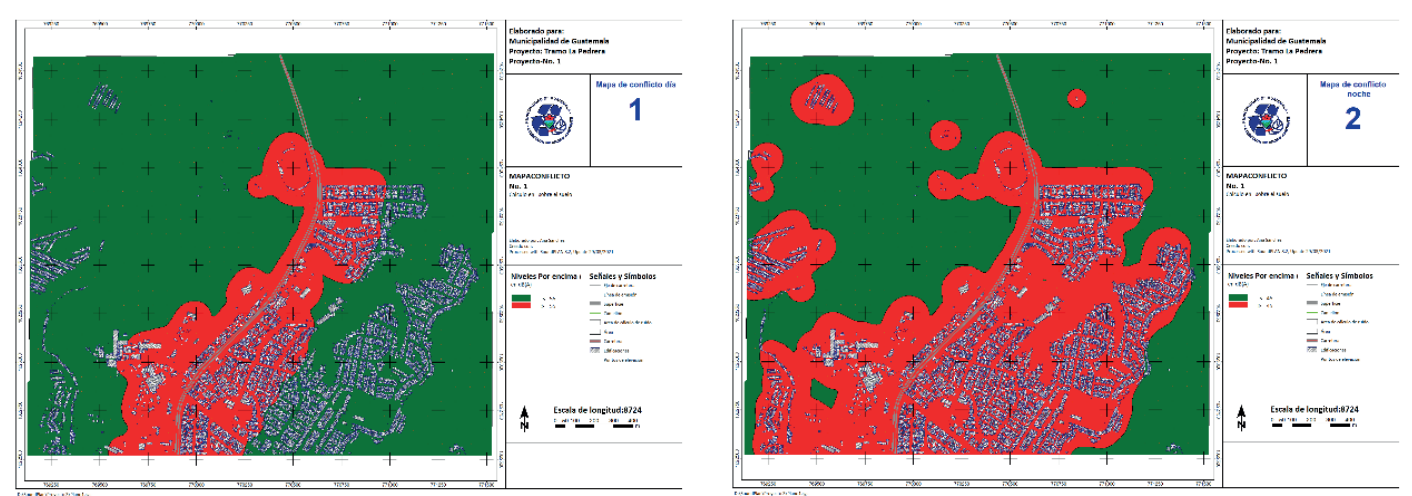

Fuente: elaboración propia, empleando SoundPLAN.

\section{Conclusiones}

SoundPLAN es un software de fácil aplicación para la generación de mapas de ruido y su representación gráfica, en el caso específico de fuentes de emisión de ruido por tránsito vehicular en carretera. Donde la normativa RLS-90 aplicada para la modelación es muy apropiada para la ciudad de Guatemala, por los datos de entrada que se requieren: edificios, área de ocupación por habitante, aforos vehiculares, elevación del terreno y características viales de los tramos.

Los resultados de los mapas de ruido para los tramos viales analizados demostraron que el flujo de tráfico, la ubicación y cantidad de edificios en el sitio incide en el valor de los niveles de ruido y cómo se distribuyen en el territorio. Resultando así que en el tramo 2 , por la alta carga vehicular, se presenten altos niveles para el periodo diurno y nocturno. $Y$ que en el tramo 3 por la concentración de edificaciones alrededor de la carretera el ruido se reduce más con la distancia afectan- do una menor cantidad de edificaciones.

De la evaluación de conflicto acústico para los tres tramos existe un incumplimiento a los niveles permitidos según la normativa española, tanto para los territorios de uso residencial (tramo 1 y 3) como aquellos de uso terciario (tramo 2). En los que para el día se superan los 65 decibeles resultando así condiciones que afectan la salud y bienestar humano por lo que deben tomarse medidas con el fin de lograr su disminución. Priorizando el tramo 2 donde los niveles llegan a ser mayores a los 100 decibeles.

La información obtenida de este estudio es de utilidad para la planificación urbana ya que permite evaluar el impacto ambiental que produce el ruido vehicular. Tanto para una situación actual como futura si se considera una nueva construcción de carreteras o la instalación de barreras acústicas, aspectos que se pueden incluir al momento de realizar 
la modelación con SoundPLAN. El arbolado urbano es un ejemplo de barrera acústica existente, no considerada en esta modelación, pero que puede influir en la reducción del ruido.

Con estos datos, además se puede establecer una línea base que sirva como referencia para la elaboración de una normativa que defina los niveles de ruido permitidos para las ciudades, tomando en cuenta las características de su territorio, y en específico para la ciudad de Guatemala, tema que debe ser considerado por la administración en el corto plazo. Para el desarrollo de la normativa se puede utilizar la normativa española, Ley española $37 / 2003$, que presenta ventaja de establecer los límites de acuerdo al uso de suelo.

\section{Agradecimientos}

A SM Tecnología por permitir el uso del programa SoundPLAN para la realización de esta modelación.

A la Dirección de Catastro y Administración del IUSI y a la Dirección de Movilidad Urbana de la Municipalidad de Guatemala por brindar la información necesaria para realizar la modelación.

\section{Referencias bibliográficas}

AACUSTICA, s.f. DESCRIPCIÓN DE MÓDULOS DE SOUNDPLAN, Vitoria-Gasteiz: AAC Centro de Acústica Aplicada, S.L.

AISTEC, s.f. Formas de ruido que pueden llevar a la elaboración de un mapa de ruido. [En línea] Disponible en: https://aistec.com/blog/fuentes-mapa-de-ruido/ acceso: 63 2021].
Campos, A., s.f. SOUNDPLAN ${ }^{\mathrm{TM}}$ - MÓDULO DE CARRETERAS, Santiago de Chile: s.n. Centro de Acústica Aplicada y Evaluación No Destructiva (CAEND), 2011. Mapas de Ruido. 27 4.p. 2.

Chaux Álvarez, L. M., 2018. Evaluación de los niveles de presión sonora (ruido ambiental) en zonas aledañas al Hospital Universitario Barrios Unidos, a la Fundación Hospital Infantil Universitario de San José, s.l.: Universidad Libre.

GeoNoise, s.f. SoundPLAN Thailand Noise Calculation and Prediction Software. [En línea] Disponible en: https://www.geonoise.com/soundplan-noise-prediction-software/ [Último acceso: 203 2021].

Gobierno de España, 2005. Ley 37/2003. s.l.: s.n.

Honour, R., 2015. Mapas de ruido, la poderosa herramienta de gestión para enfrentar el ruido en las ciudades. 284 .

Mishra, A. R., Mishra, R. K. \& Singh, A., 2019. TRAFFIC NOISE ANALYSIS USING RLS-90 MODEL IN URBAN CITY, Delhi: inter.noise.

Organización Mundial de la Salud (OMS), 1999. Guidelines for Community Noise, s.l.: s.n.

PARLAMENTO EUROPEO Y CONSEJO DE LA UNIÓN, 2002. DIRECTIVA 2002/49/CE sobre evaluación y gestión del ruido ambiental. [En línea] Disponible en: http://sicaweb.cedex.es/docs/leyes/Directi$\mathrm{v} a-2002-49-\mathrm{C} E-\mathrm{E} v \mathrm{a}$ luacion-gestion-ruido-ambiental.pdf

Peña Gutiérrez, J. C., 2012. Bogotá: s.n.

Pitán, E., 2015. Contaminación auditiva en el país. 222. 
Rodríguez Casals, C., 2016. El problema de la contaminación acústica en nuestras ciudades, s.l.: s.n.
SoundPLAN, s.f. Facade Noise Map. [En línea] Disponible en: http://www.soundplan.net/index.php/grid-noise-map [Último acceso: 23 2021].

\section{Sobre autor}

\section{Ana Isabel Sánchez Girón}

Ingeniera ambiental de la Universidad de San Carlos de Guatemala (USAC). Maestrante en Gestión y Auditorías Ambientales por la Universidad de San Carlos de Guatemala (USAC) y la Universidad Internacional Iberoamericana (UNINI-México). Técnico Ambiental en la Dirección de Medio Ambiente de la Municipalidad de Guatemala. Integrante del comité técnico de normalización COGUANOR para la norma de Ruido Ambiental.

\section{Sobre autor}

\section{Marco Antonio Urrutia Campo}

Ingeniero ambiental egresado de la Universidad de San Carlos de Guatemala (USAC). Maestrante en Estadística Aplicada por la Universidad de San Carlos de Guatemala. Coordinador de la Oficina forestal Municipal de la ciudad de Guatemala. Miembro del comité técnico de normalización COGUANOR para la norma de Ruido Ambiental. Miembro de la Mesa de Vulnerabilidad, AVE.

Copyright (c) 2021 Ana Isabel Sánchez Girón y Marco Antonio Urrutia Campo

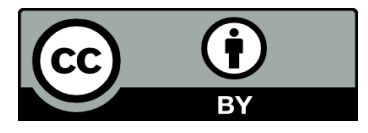

Este texto está protegido por una licencia $\underline{\text { Creative Commons }} \underline{4.0}$.

Usted es libre para Compartir — copiar y redistribuir el material en cualquier medio o formato- y Adaptar el documento remezclar, transformar y crear a partir del material - para cualquier propósito, incluso para fines comerciales, siempre que cumpla la condición de:

Atribución: Usted debe dar crédito a la obra original de manera adecuada, proporcionar un enlace a la licencia, e indicar si se han realizado cambios. Puede hacerlo en cualquier forma razonable, pero no de forma tal que sugiera que tiene el apoyo del licenciante o lo recibe por el uso que hace de la obra.

\section{$\underline{\text { Resumen delicencia - Textocompletodelalicencia }}$}

Revista digital: ISSN 2707-9643

Revista impresa: ISSN 2313-786X

Editorial Servi Prensa, Guatemala

https://doi.org/10.37533/cunsurori.v8i1.66
Vol. 8 No. 1

Enero - Diciembre 
